\title{
THE COMPLEXIFICATION AND DIFFERENTIAL STRUCTURE OF A LOCALLY COMPACT GROUP
}

\author{
BY
}

\author{
KELLY MCKENNON
}

\begin{abstract}
The concept of a complexification of a locally compact group is defined and its connections with the differential structure developed. To provide an interpretation in terms of irreducible representations of separable, Type I groups, a duality theorem and Bochner theorem are presented.
\end{abstract}

The Pontryagin-Van Kampen duality theorem states that any locally compact Abelian group may be regarded as the set of continuous homomorphisms on a dual LCA group $X$ into the circle group $T$. Thus $G$ is always a subgroup of the group $G_{C}$ of continuous homomorphisms of $X$ into the multiplicative group of nonzero complex numbers. It is not difficult to see that $G_{C}$ is the direct product of $G$ and the group $G_{C}^{+}$of continuous homomorphisms of $X$ into the multiplicative group of positive real numbers. The natural logarithm converts $G_{C}^{+}$into the linear space of continuous real homomorphisms of $X$, which space in turn is transformed via the map $f \rightarrow e^{-i f}$ into the Lie algebra $\Lambda$ of continuous one-parameter subgroups of $G$ (see [11, 24.33 et seq.]). Thus the "complexification" $G_{C}$ may be viewed as a product of $G$ with its Lie algebra $\Lambda$. Since the component of the identity contains all the one-parameter subgroups, it is convenient to deal only with connected groups when studying the complexification.

The situation for a compact group is similar, though it must be couched in terms of the algebra $T(G)$ of trigonometric polynomials (or representative functions) on $G$ and relies on Tannaka's duality theorem [13]. The dual of $T(G)$ may be regarded as a product $M$ of simple finite-dimensional von Neumann algebras and Tannaka's theorem states that the unitary elements of this product which are in the spectrum of the multiplicative algebra $T(G)$ may be identified with $G$. Thus $G$ is a subset of the spectrum $G_{C}$ of $T(G)$. If $G_{C}^{+}$denotes the elements of $G_{C}$ which are positive in $M$, then $G_{C}^{+}$is, in general, not a group, but it is still true that $G_{C}$ is a group and is the direct product of $G$ with $G_{C}^{+}$. The function $i \ln$ (viewed as a function on the product $M$ of von Neumann algebras) maps $G_{C}^{+}$onto the algebra of derivations of $T(G)$ and the map $x \rightarrow e^{t x}$ transform the Lie algebra of derivations onto the Lie algebra of one-parameter subgroups of $G$ (see [6] for Lie groups and [18] for the general case).

It is the business of the sequel to investigate to what extent the idea of a "complexification" and its connection with the differential structure extends to a

Received by the editors May 14, 1979 and, in revised form, June 30, 1980.

1980 Mathematics Subject Classification. Primary 43A40; Secondary 22E65, 32A15, 32D25, 46L05.

(C) 1981 American Mathematical Society 0002-9947/81/0000-0416/\$06.50 
general locally compact group. The task is complicated by the fact that a perfectly satisfactory generalization of the Pontryagin-Van Kampen theory does not seem to be available for general locally compact groups, although there have been many attempts. A number of these, closely connected with the left regular representation of $G$, have been unified by Takesaki [23] in a theory based on Hopf-von Neumann algebras. We have chosen here to clad our ideas in the trappings of the group $W^{*}$-algebras, and so will have recourse to the theory of Walter [25].

We begin in $\$ 1$ with some technical matters allowing us to speak of "unbounded" elements of a $W^{*}$-algebra. In $\$ 2$ the group complexification $G_{C}$ is introduced and its basic structure determined. $\$ 3$ presents the relation $G_{C}$ has to the group Lie algebra. The unboundedness of the positive elements of $G_{C}$ (the elements of $R_{C}^{+}$, for instance, are the exponential functions on $R$ ) causes a problem in considering $G_{C}$ as a group, which is discussed in $\S 4$. The remainder of the paper is concerned with the complexification relative to various representations-particularly the irreducible ones. The principal tools here are a Bochner-type theorem (\$7) and a duality theorem $(\$ 8)$.

Since the reader may find the symbols employed in this paper somewhat formidable in number, a list is provided immediately preceding the bibliography. A number $x^{+}\left(x^{-}\right)$after a listing means that that particular symbol is first found just after (before) the number $(x)$ in the text.

1. Background. Let $W$ be a $W^{*}$-algebra with identity $\iota$, adjoint $\sim$, and multiplication * . We shall view $W$ as a family of linear functionals on its predual $W_{*}$. The positive cones of $W$ and $W_{*}$ will be denoted by $W^{+}$and $W_{*}^{+}$, respectively. For $\omega \in W$ and $F \in W_{*}$ we define $\omega * F, F * \omega \in W_{*}$ by letting $\omega * F(\theta) \equiv$ $F\left(\omega^{\sim} * \theta\right)$ and $F * \omega(\theta) \equiv F\left(\theta * \omega^{\sim}\right)$ for all $\theta \in W$.

By a resolution of a projection $\pi_{\infty}$ in $W$ we shall mean a map $\pi$ from the family $B$ of Borel subsets of a locally compact Hausdorff space $X(\pi)$ into the set of projections in $W^{+}$such that:

(1a) $\pi_{\phi}=0, \pi_{X(\pi)}=\pi_{\infty}$

(lb) $\pi_{Y} \neq 0(\forall Y \subset X(\pi): Y$ is nonvoid and open);

(lc) $\pi_{Y \cap Z}=\pi_{Y} \pi_{Z}(\forall Y, Z \in B)$;

(1d) $\pi_{Y \cup Z}=\pi_{Y}+\pi_{Z}(\forall Y, Z \in B: Y \cap Z=\varnothing)$;

(le) $\mu(\pi, F) \mid B \ni Y \rightarrow \pi_{Y}(F)$ is a regular complex measure for all $F \in W_{*}$.

By a resolution of a partial unitary element $\nu_{\infty}$ of $W$, we shall mean a map $\nu$ from the Borel $\sigma$-algebra $B$ of a locally compact space $X(v)$ into the partial unitary elements of $W$ such that $\nu_{\infty}=\nu_{X}$ and $\nu^{\sim} * \nu$ is a resolution of the projection

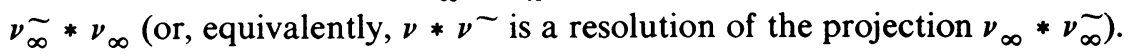

Let $R=R(W)$ be the family of all resolutions $\nu$ of partial unitary elements such that $X(\nu)$ is a closed subset of the set $\mathbf{R}^{+}$of positive real numbers, and write $R^{+}$ for the set of resolutions of projections in $R$. For $\nu \in R$, let $W_{*}^{+}[\nu]$ be the order ideal consistinig of all $F \in W_{*}^{+}$such that

$$
\int_{X(\nu)} x^{2} d \mu\left(\nu^{\sim * \nu}, F\right)(x)<\infty
$$


it is a consequence of the spectral theory that $W_{*}^{+}[\nu]$ is dense in $W_{*}^{+}$. Thus the linear span $W_{*}[\nu]$ of $W_{*}^{+}[\nu]$ is dense in $W_{*}$. For each $\nu \in R$ define $\nu^{-} \mid W_{*}[\nu] \rightarrow C$ by

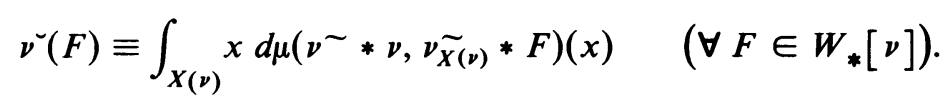

The existence of (3) is justified by the fact that if $F \in W_{*}^{+}[\nu]$ and $S$ is any $W^{*}$-representation of $W$ on a Hilbert space $H$ with a vector $v \in H$ satisfying $\left\langle S_{\omega} v, v\right\rangle=\omega(F)$ for all $\omega \in W$, the spectral theory guarantees the existence of a unique positive selfadjoint operator

$$
A=\int x d S_{\nu \sim, \nu}(x)
$$

and thus (2) becomes $\langle A v, A v\rangle<\infty$, which implies that

$$
\int_{X(\nu)} x d \mu\left(\nu \sim * \nu, \tilde{\nu_{X}(\nu)} * F\right)(x)=\left\langle A v, S_{\nu_{X(\nu)}}^{\sim} v\right\rangle
$$

is well defined.

Define $\bar{W}$ to be the set of all $\nu^{\smile}$ such that $\nu \in R$, and write $\operatorname{dom}(\nu)$ for its domain $W_{*}[\nu]$. Let $\nu \in R$ be arbitrary, and consider any $W^{*}$-representation $S$ of $W$ on a Hilbert space $H$. Let $A$ satisfy (4) and write $\nu_{\infty}$ for $\nu_{X(v)}$. Then $S_{\nu_{\infty}} \circ A$ is a closed linear operator on $H$ and we define

$$
S_{\nu^{-}} \equiv S_{\nu_{\infty}} \circ A
$$

Note further that, if $S(W)^{\prime}$ denotes the commutant of $S(W)$, then

$$
S_{\nu^{-}} \circ V=V \circ S_{\nu} \quad\left(\forall V \in S(W)^{\prime}\right) .
$$

On the other hand, if $R$ is a closed linear operator with dense domain, it can be written uniquely as a product $U \circ A$ where $A$ is positive selfadjoint, $U$ is a partial unitary operator, and $R, A$ and $U$ have identical initial projections [7, XII 7.7]; if $R \circ V=V \circ R$ as well for all $V \in S(W)^{\prime}$, then the same holds for $A$ and $U$ so $U \in S(W)$ and $A=\int x d P$, where $P$ is a resolution of the initial projection of $A$ in the $W^{*}$-algebra $S(W)$. Thus if

$$
W=W(S) \oplus \operatorname{ker}(S)
$$

is the canonical decomposition of $W$ into a direct sum of ideals,

$$
(\exists ! \nu \in R(W(S))) \quad S_{\nu}=R .
$$

When $S$ is faithful on $W$, it follows from (7) that its extension to $\bar{W}$ is also faithful; hence we can define

$$
\theta * \omega \equiv S^{-1}\left(S_{\theta} \circ S_{\omega}\right) \quad(\forall \theta \in W, \omega \in \bar{W})
$$

and a simple approximation argument shows that the definition is independent of the particular faithful $S$ employed.

For $\omega \in \bar{W}$ and $F \in \operatorname{dom}(\omega)$, we define

$$
\omega * F \mid W \ni \theta \rightarrow \omega^{\sim} * \theta(F) \text {. }
$$


We leave it to the reader to verify that, if $S$ is a $W^{*}$-representation on $H$, then $(\forall \omega, \theta \in \bar{W})$,

$$
S_{\omega}+S_{\theta} \text { is densely defined if } \operatorname{dom}(\omega) \cap \operatorname{dom}(\theta) \text { is dense in } W_{*},
$$

and

$S_{\omega} \circ S_{\theta}$ is densely defined if $\left\{\theta^{\sim} * F: F \in \operatorname{dom}(\theta)\right\} \cap \operatorname{dom}(\omega)$ is dense in $W_{*}$,

and the converse holds if $\omega, \theta \in \overline{W(S)}$. Thus, for $\omega, \theta \in \bar{W}, \omega+\theta$ is defined and satisfies

$$
(\omega+\theta)(F)=\theta(F)+\omega(F) \quad(\forall F \in \operatorname{dom}(\theta) \cap \operatorname{dom}(\omega))
$$

whenever $\operatorname{dom}(\theta) \cap \operatorname{dom}(\omega)$ is dense in $W_{*}$, and

$$
S_{\omega+\theta}=S_{\omega}+S_{\theta} \text {. }
$$

Likewise $\omega * \theta$ is defined and satisfies

$$
\omega * \theta(F)=\omega(\theta \sim F) \quad(\forall F \in \operatorname{dom}(\theta))
$$

whenever $\left\{\theta^{\sim} * F: F \in \operatorname{dom}(\theta)\right\} \cap \operatorname{dom}(\omega)$ is dense in $W_{*}$, and

$$
S_{\omega * \theta}=S_{\omega} \circ S_{\theta} \text {. }
$$

The adjoint can analogously be extended to $\bar{W}$ such that

$$
S_{\omega \sim}=S_{\omega}^{\sim} \quad(\forall \omega \in \bar{W}) .
$$

If $\omega \in \bar{W}$ is selfadjoint and $f$ is any continuous complex-valued function on the spectrum of $\omega$, one can define (where $S$ is any faithful $W^{*}$-representation)

$$
f(\omega) \equiv S^{-1}\left(f\left(S_{\omega}\right)\right) \text {. }
$$

We close the present section by noting that, if $\psi \mid W \rightarrow A$ is any homomorphism of $W^{*}$-algebras, $\psi$ has a canonical extension (also denoted $\psi$ ) of $\bar{W}$ into $\bar{A}$ such that

$$
f(\psi(\omega))=\psi(f(\omega))
$$

where $\omega \in \bar{W}$ is selfadjoint and $f$ is any continuous complex-valued function on the spectrum of $\omega$.

2. The complexification of a group. Let $G$ be a locally compact group, $e$ its identity, $\lambda$ a left Haar measure on $G, L^{1}=L^{1}(G, \lambda)$ the Banach *-algebra of $\lambda$-integrable functions, $\mathcal{C}=\mathcal{C}(G)$ the enveloping $C^{*}$-algebra of $G, W=W(G)$ the enveloping $W^{*}$-algebra of $C, C=C(G)$ the algebra of continuous complex-valued functions on $G, P=P(G)$ the cone of positive definite elements of $C$, and $B=B(G)$ the linear span of $P$ in $C$. Then $B(G)$ is the Fourier-Stieltjes algebra of $G$ and may be associated with $W_{*}$ via a canonical map $F \mid B \rightarrow W_{*}$ satisfying

$$
h\left(F_{f}\right)=\int_{G} h f d \lambda \quad\left(\forall h \in L^{1}, f \in B\right)
$$

(where we view $L^{1} \subset C \subset W$ ). For $\omega \in \bar{W}$ and $F_{f} \in W_{*}[\omega]$, we define

$$
\langle\omega, f\rangle \equiv \omega\left(F_{f}\right)
$$


Let $\gamma$ be the canonical monomorphism of $G$ into the group of unitary elements of $W$-then

$$
\langle\gamma(x), f\rangle=f(x) \quad(\forall x \in G, f \in B) .
$$

We shall frequently use the fact that the continuous unitary representations of $G$ and $C^{*}$-algebra representations of $C$ are, respectively, just the representations $V \circ \gamma$ and $\left.V\right|_{C}$, where $V$ runs through the class of $W^{*}$-algebra representations of $W$ (here a $C^{*}$-algebra representation implies norm-continuity and a $W^{*}$-algebra representation means weak ${ }^{*}$-continuity) $[8,12.1 .5$ and 13.9.3].

To each $f \in P(G)$ is associated a unique (up to unitary equivalence) cyclic $W^{*}$-algebra representation $T^{f}$ of $W$ on a Hilbert space $H(f)$ satisfying

$$
\left\langle T_{\omega}^{f} v_{f}, v_{f}\right\rangle_{f}=\langle\omega, f\rangle \quad(\forall \omega \in W)
$$

where $\langle,\rangle_{f}$ and $v_{n}$ are the inner product and cyclic vector, respectively, in $H(f)$. For $f$ and $h$ in $P$, the tensor product unitary representation $T^{f} \circ \gamma \otimes T^{h} \circ \gamma$ of $G$ is connected with a unique $W^{*}$-representation $T^{f, h}$ of $W$ such that

$$
T_{\gamma(x)}^{f} \otimes T_{\gamma(x)}^{h} h=T_{\gamma(x)}^{f, h} \quad(\forall x \in G) .
$$

By the complexification $G_{\mathbf{C}}$ of $G$ we shall mean the set of all $\omega \in \bar{W}$ such that

$$
T_{\omega}^{f} \otimes T_{\omega}^{h}=T_{\omega}^{f, h} \quad(\forall f, h \in P) .
$$

It trivially follows from (19) that the image $G_{\gamma}$ of $G$ by $\gamma$ is a subset of $G_{\mathrm{C}}$. We write $G_{\mathbf{C}}^{+}$for $G_{\mathbf{C}} \cap \bar{W}^{+}$. In dealing with $G_{\mathbf{C}}^{+}$it is convenient to introduce the set $\Lambda=\Lambda(G)$ of all $\alpha \in \bar{W}$ such that

$$
T_{\alpha}^{f} \otimes I+I \otimes T_{\alpha}^{h}=T_{\alpha}^{f, h} \quad(\forall f, h \in P)
$$

(where $I$ always represents the identity operator) and

$$
\alpha^{\sim}=-\alpha \text {. }
$$

Proposition 1. For $\alpha \in \Lambda$ and $z \in \mathbf{C}$, $\exp (z \alpha)$ is in $G_{\mathbf{C}}$.

Proof. Let $f, h \in P$ be arbitrary. That $T_{\exp (z \alpha)}^{f, h}=\exp z T_{\alpha}^{f, h}$ is trivial. That

$$
T_{\exp (z \alpha)}^{f} \otimes T_{\exp (z \alpha)}^{h}=\exp z\left(T_{\alpha}^{f} \otimes I+I \otimes T_{\alpha}^{f}\right)
$$

follows from an elementary rearrangement of power series. Thus (21) and (23) imply

$$
T_{\exp (z \alpha)}^{f} \otimes T_{\exp (z \alpha)}^{h}=T_{\exp (z \alpha)}^{f, h} \text {. Q.E.D. }
$$

Proposition 2. The map $\Lambda \ni \alpha \rightarrow \exp (-i \alpha)$ is a bijection onto $G_{\mathbf{C}}^{+}$.

Proof. That the map under consideration is injective is a consequence of the spectral theory. In view of (22) and Proposition 1, it remains only to show the map is surjective.

Let $\pi \in G_{\mathbf{C}}^{+}$and $f, h \in P$ be arbitrary. For each $n \in \mathbf{N}$, define $f_{n} \mid \mathbf{R} \ni x \rightarrow$ $\max \left\{n^{-1}, \min (n, x)\right\}$ and choose $\pi_{n} \in \bar{W}^{+}$such that $f_{n}(\pi)=\pi_{n}$. Then the series

$$
\ln (n \iota)+\sum_{m=1}^{\infty}(-1)^{m+1} m^{-1} n^{-m}\left(\pi_{n}-n \iota\right)^{m}
$$


converges absolutely to $\ln \pi_{n}$. A simple exercise with power series now leads to

$$
\ln \left(T_{\pi_{n}}^{f} \otimes T_{\pi_{n}}^{h}\right)=\left(\ln T_{\pi}^{f} \otimes I\right)+\left(I \otimes \ln T_{\pi_{n}}^{h}\right) .
$$

Since the sequence $T_{\pi_{n}}^{f}\left(T_{\pi_{n}}^{h}\right.$, resp.) converges to $T_{\pi}^{f}\left(T_{\pi}^{h}\right.$, resp.) on a dense subset of $H(f)(H(h)$, resp.), (24) implies

$$
\ln \left(T_{\pi}^{f} \otimes T_{\pi}^{h}\right)=\left(\ln T_{\pi}^{f} \otimes I\right)+\left(I \otimes \ln T_{\pi}^{h}\right) .
$$

But $\pi$ is in $G_{\mathrm{C}}^{+}$so, if $\alpha \equiv-i \ln \pi$, (25) implies that (21) holds. That (22) holds follows from the spectral theory. Hence $\alpha \in \Lambda$ and $\exp (-i \alpha)=\pi$. Q.E.D.

Proposition 3. For $\omega \in G_{\mathbf{C}}$, both $\omega^{-1}$ and $\omega^{\sim}$ are in $G_{\mathbf{C}}$. If $\omega, \beta \in G_{\mathbf{C}}$ and $\omega * \beta$ is defined in $\bar{W}$, then $\omega * \beta$ is in $G_{\mathbf{C}}$.

Proof. Let $f, h \in P$ be arbitrary. Then

$$
T_{\omega^{-1}}^{f} \otimes T_{\omega^{-1}}^{h}=\left(T_{\omega}^{f} \otimes T_{\omega}^{h}\right)^{-1}=\left(T_{\omega}^{f, h}\right)^{-1}=T_{\omega^{-1}}^{f, h},
$$

so $\omega^{-1}$ is in $G_{\mathbf{C}}$. That $\omega^{\sim}$ is in $G_{\mathbf{C}}$ is proved analogously. If $\omega, \beta \in G_{\mathbf{C}}$ and $\omega * \beta$ is defined, then

$$
T_{\omega \bullet \beta}^{f} \otimes T_{\omega \bullet \beta}^{h}=\left(T_{\omega}^{f} \otimes T_{\omega}^{h}\right) \circ\left(T_{\beta}^{f} \otimes T_{\beta}^{h}\right)=T_{\omega}^{f, h} \circ T_{\beta}^{f, h}=T_{\omega \bullet \beta}^{f, h},
$$

so $\omega * \beta$ is in $G_{\mathbf{C}}$. Q.E.D.

TheOREM 1. The product $G_{y} * G_{\mathrm{C}}^{+}$is direct and equals $G_{\mathrm{C}}$.

Proof. That $G_{\gamma} * G_{\mathrm{C}}^{+} \subset G_{\mathrm{C}}$ follows from Proposition 3. That $G_{\gamma} * G_{\mathrm{C}}^{+}$is direct follows from the uniqueness of polar decomposition of invertible operators.

Let $\omega \in G_{\mathrm{C}}$ be arbitrary and write $\nu * \pi$ for its polar decomposition, $\nu \in W$ unitary and $\pi \in \bar{W}^{+}$. Proposition 3 implies that $\omega^{\sim}$ is in $G_{\mathrm{C}}$ and that $\omega^{\sim} * \omega=\pi^{2}$ is in $G_{\mathbf{C}}^{+}$. Proposition 2 yields $\alpha \in \Lambda$ such that $\exp (-i \alpha)=\pi^{2}$. Proposition 1 now implies that $\exp (-i \alpha / 2)=\pi$ and $\exp (i \alpha / 2)=\pi^{-1}$ are in $G_{\mathbf{C}}^{+}$. Hence, by Proposition 3 again $\nu=\omega * \pi^{-1}$ is in $G_{\mathrm{C}}$. But Walter's duality theorem [25, Theorem 1] states that $G_{\gamma}$ is precisely the family of unitary elements of $G_{\mathbf{C}}$; hence $\omega \in$ $G_{\gamma} * G_{\mathbf{C}}^{+}$. Q.E.D.

3. The complexification and the group Lie algebra. Thus far we have omitted discussing if or when $G_{C}$ is a group. This question is connected with the differential structure on $G$, which we shall discuss in the present section. We shall denote by $T$ the direct sum of all representations $T^{f}, f \in P$, and by $H(T)$ the representation space.

Let $\Gamma=\Gamma(G)$ be the family of continuous one-parameter subgroups of $G$. For $\alpha \in \Lambda$, the map $s^{\alpha} \mid \mathbf{R} \ni t \rightarrow \gamma^{-1}(\exp (t \alpha))$ is evidently a one-parameter subgroup of $G$.

Proposition 4. The map $\Lambda \ni \alpha \rightarrow s^{\alpha}$ is a bijection onto $\Gamma$.

Proof. It follows from spectral theory that there is a dense subspace $M$ of $H$ on which all the operators $T_{\exp (t \alpha)}$ are defined and such that

$$
\lim _{t \rightarrow 0} T_{\exp (t \alpha)}=I \text { pointwise on } M \text {. }
$$


Since the operators $T_{\exp (t \alpha)}$ are all unitary, the convergence (26) holds pointwise on all of $H(T)$. Since $\gamma$ is a homeomorphism [25, Theorem 1] when $W$ carries the weak-* topology $\sigma\left(W, W_{*}\right)$, it follows that $\lim _{t \rightarrow 0} \gamma^{-1}(\exp (t \alpha))=e$ in $G$. Hence $s^{\alpha} \in \Gamma$ for each $\alpha \in \Lambda$.

The formula

$$
\lim _{t \rightarrow r}(t-r)^{-1}\left(T_{\exp (t \alpha)}-T_{\exp (r \alpha)}(v)\right)=T_{\alpha} \circ T_{\exp (r \alpha)}(v)
$$

is valid for all $r \in \mathbf{R}$ and $v$ in the domain of $T_{\alpha}$ [22, 13.35]. When $r=0$ (27) implies that $\Lambda \ni \alpha \rightarrow s^{\alpha}$ is injective.

Now suppose that $s \in \Gamma$ is arbitrary. Then the map $\mathbf{R} \ni t \rightarrow T_{\gamma(s(t))}$ is a continuous, one-parameter subgroup of unitary operators so, by Stone's theorem [22, 13.37], there exists a closed skew operator $V$ on $H(T)$ such that $T_{\gamma(s(t))}=\exp (t V)$ for all $t \in \mathbf{R}$. Since $V$ evidently commutes with each element of $T(W)^{\prime}$, we have $V=T_{\alpha}$ for some $\alpha \in \bar{W}$ satisfying (22). Since $\lim _{t \rightarrow 0} t^{-1}[\exp (t V)(v)-v]=V(v)$ for all $v$ in a dense subspace of $H(T)$, the product rule shows that (21) holds. Hence $\alpha \in \Lambda$ and $s=s^{\alpha}$. Q.E.D.

Since the range of each $s \in \Gamma$ is connected, it is contained in the component of $e$ in $G$. Thus, in view of Propositions 2 and 4, we lose little when studying $G_{\mathbf{C}}$ by considering only connected groups.

Throughout the sequel, $G$ will always be assumed to be connected.

Combining ideas of Mackey [16], Riss [21], and Bruhat [5], Boseck and Czichowski [3], [4] have constructed a theory of differentiability for $G$ based on $\Gamma$.

Let $E$ be a complete, locally convex, continuous $G$-module with dual $E^{\prime}$. A vector $v \in E$ is said to be differentiable if

$$
D_{s} v \equiv \sigma\left(E, E^{\prime}\right)-\lim _{t \rightarrow 0} \frac{s(t) \cdot v-v}{t} \text { exists in } E \quad(\forall s \in \Gamma) \text {; }
$$

the set of all $v \in E$ such that $D_{s} D_{r} \ldots D_{q} v$ is differentiable for every finite subset $\{s, r, \ldots, q\}$ of $\Gamma$ will be denoted $E_{\infty}$. An important subset $E_{\infty, 1}$ consists of those vectors $v \in E_{\infty}$ such that the stationary subgoup $\{x \in G: x \cdot v=0\}$ contains a compact normal subgroup $S_{v}$ of $G$ such that $G / S_{v}$ is a Lie group. The BoseckGårding theorem [4, Satz 3.1] states that

(29) $E_{\infty, 1}$ is dense in $E$.

If $E$ contains a topologically $G$-cyclic vector $v \in E_{\infty, 1}$, then the normality of $S_{v}$ implies its containment in the annihilator $G(E)$ of $E$, which yields

(30) $G / G(E)$ is a Lie group.

Boseck's work is based on Yamabe's theorem [28] that there exists a directed family $\mathcal{G}$ of compact normal subgroups such that each quotient $G / N, N \in \mathcal{G}$, is a Lie group and

(31) $G$ is topologically isomorphic to proj $\lim _{N \in \mathcal{G}} G / N$.

Lashof [15] used (31) to construct a group Lie algebra for $G$, which he defined as the inductive (or inverse) limit of the Lie algebras of the Lie groups $G / N$. Boseck 
showed that Lashof's Lie algebra is isomorphic with $\Gamma$ [4, Hilfsatz 2.2] when one defines

$$
\begin{gathered}
r \cdot s|\mathbf{R} \ni t \rightarrow s(r t), \quad s+w| \mathbf{R} \ni t \rightarrow \lim _{n \rightarrow \infty}\left(s\left(\frac{t}{n}\right) w\left(\frac{t}{n}\right)\right)^{n}, \\
{[s, w] \mid R \ni t^{2} \rightarrow \lim _{n \rightarrow \infty}\left(s\left(\frac{-t}{n}\right) w\left(\frac{-t}{n}\right) s\left(\frac{t}{n}\right) w\left(\frac{t}{n}\right)\right)^{n^{2}},} \\
{[s, w] \mid R \ni-t^{2} \rightarrow[w, s]\left(t^{2}\right) .}
\end{gathered}
$$

Proposition 4 and (27) imply that $H(T)_{\infty}$ is an invariant subset of the domain of each operator $T_{\alpha}, \alpha \in \Lambda$. Thus sums and products of elements of $\Lambda$ are well defined, and evidently it is a Lie algebra. It is not difficult to see that the map $\Lambda \ni \alpha \rightarrow s^{\alpha} \in \Gamma$ is in fact a Lie algebra isomorphism. Hence we do not essentially conflict with Lashof in calling $\Lambda$ the group Lie algebra. If $g \mid G_{1} \rightarrow G_{2}$ is a continuous homomorphism of connected groups, the map $\Gamma\left(G_{1}\right) \ni s \rightarrow g \circ s \in \Gamma\left(G_{2}\right)$ induces a Lie algebra homomorphism of $\Lambda\left(G_{1}\right)$ into $\Lambda\left(G_{2}\right)$. When $g$ is an epimorphism and $G$ is a Lie group, a simple dimension argument shows that the Lie algebra homomorphism is surjective as well. Our next task is to show this still holds for general connected $G_{1}$, which is modest generalization of [19, 4.15].

Lемма 1. Let $\mathcal{D}$ be a directed family of Lie groups and suppose that, for all $H, S \in \mathscr{D}$ with $H>S$, there is given a continuous epimorphism $\varepsilon_{H, S}$ of $H$ on $S$. If $E \subset \mathscr{D}$ and $s \in \Gamma\left(\mathrm{II}_{H \in E} H\right)$, say that $(E, s)$ satisfies property $\mathrm{P}$ if, for any finite subset $F$ of $E$, there exist $H \in \mathcal{D}$ and $r \in \Gamma(H)$ such that $H>S$ and $E_{H, S} \circ r=s_{S}$ for all $S \in F$. Then, if a pair $(E, s)$ does satisfy property $\mathrm{P}$, there exists $q \in$ $\Gamma\left(\right.$ proj $\left.\lim _{H \in D} H\right)$ such that $q(t)_{H}=s(t)_{H}$ for all $t \in \mathbf{R}$ and $H \in E$.

Proof. For each $H \in \mathscr{D}$, let $\Lambda(H)$ be the Lie algebra of $H$. Well-order $\mathscr{D}$ such that $F$ is an initial section $I(E)$ and write $<$ for this well-ordering. Suppose that $(I(A), q)$ satisfies property $\mathrm{P}$ (where $I(A)$ is an initial section determined by some $A \in \mathscr{D})$ and $q(t)_{H}=s(t)_{H}$ for all $t \in R$ and $H \in E \cap I(A)$. If $A \in E$, let $q(t)_{A} \equiv s(t)_{A}$ for all $t \in R$-if $A \notin E$, proceed as follows: let $\Phi$ be the family of all finite subsets of $I(E)$ and, for each $F \in \Phi$, define $\mho(F)$ to be the union of all sets

$$
d \varepsilon_{D, A}\left(\left\{\theta \in \Lambda\left(D: \exp _{B}\left(d \varepsilon_{D, B}(t \theta)\right)=q(t)_{B}(\forall B \in \Phi, t \in \mathbf{R})\right)\right\}\right)
$$

where $D \in \mathscr{D}$ satisfies $D>S$ for all $S \in F \cup\{A\}$ (here $d \varepsilon_{D, A}$ is the differential of $\varepsilon_{D, A}$ and $\exp _{B}$ the exponential from the Lie algebra $\Lambda(B)$ into $B$ ). Since $\mathscr{D}$ is directed and $(I(A) ; q)$ satisfies property $\mathrm{P}$, it follows that each $\mathscr{W}(F)$ is nonvoid. Further, since each $\mathscr{W}(F)$ is an affine subset of the finite-dimensional space $\Lambda(A)$, there exists at least one element $\eta$ in the intersection of them all. Let $q(t)_{A} \equiv$ $\exp _{A}(t \eta)$ for all $t \in \mathbf{R}$, and regard now $q$ as an element of $\Gamma\left(\Pi_{H \in I(A) \cup\{A\}} H\right)$. Evidently $(I(A) \cup\{A\}, q)$ satisfies property P. It now follows from the Principle of Transfinite Induction that $q$ can be extended to an element (which we also denote by $q)$ of $\Gamma\left(\Pi_{H \in D} H\right)$ such that $(\mathscr{D}, q)$ has property $P$. That $q$ is in $\Gamma\left(\right.$ proj $\left.\lim _{H \in \mathscr{D}} H\right)$ is clear. Q.E.D. 
THEOREM 2. For the locally compact groups $G$ and $H$, let $w \mid G \rightarrow H$ be a continuous homomorphism and $s \in \Gamma(H)$ satisfy $s(\mathbf{R}) \subset w(G)$. Then there exists $r \in \Gamma(G)$ such that $w \circ r=s$.

Proof. The component $H_{c}$ of the identity in $H$ contains $s(\mathbf{R})$ and is topologically isomorphic to proj $\lim _{K \in K(H)} H_{c} / K$ where $K(H)$ is the family of normal compact subgroups $K$ of $H$ such that $H_{c} / K$ is a Lie group. If $\pi_{K} \mid H_{c} \rightarrow H_{c} / K$ is the quotient map for each $K \in K(H)$, then the canonical homomorphisms

$$
\rho_{K} \mid G_{c} /\left[G_{c} \cap \operatorname{Ker}\left(\pi_{K} \circ w\right)\right] \rightarrow H_{c} / K
$$

are injective and continuous-hence the domain of each $\rho_{K}$ is a Lie group [14, VIII.1.1], and each $\rho_{K}^{-1} \circ \pi_{K} \circ s$ is in $\Gamma\left(G_{c} /\left[G_{c} \cap \operatorname{Ker}\left(\pi_{K} \circ w\right)\right]\right)$ [14, X.4.1]. Let OD $\equiv\left\{G_{c} / S: S\right.$ is a closed normal subgroup of $G_{c}$ and $G_{c} / S$ a Lie group $\}$ be a directed set under the natural direction, let

$$
\mathcal{E} \equiv\left\{G_{c} /\left[G_{c} \cap \operatorname{Ker}\left(\pi_{K} \circ w\right)\right]: K \in K(H)\right\}
$$

and define $\bar{r} \in \Gamma\left(\Pi_{W \in E} W\right)$ by letting

$$
\bar{r}(t)_{G_{c} /\left[G_{c} \cap \operatorname{Ker}\left(\pi_{K} \circ w\right)\right]} \equiv \rho_{K}^{-1} \circ \pi_{K} \circ s
$$

for each $K \in K(H)$. Evidently, $(\mathcal{E}, \vec{r})$ satisfies property $\mathrm{P}$ of the preceding lemma. Thus $\bar{r}$ has an extension (which we also write as $\bar{r}$ ) to an element of $\Gamma\left(\operatorname{proj} \lim _{S \in \mathcal{D}} G_{c} / S\right)$. If $v \mid G_{c} \rightarrow \operatorname{proj} \lim _{S \in \mathscr{D}} G_{c} / S$ is the canonical topological isomorphism, then the map $r \equiv v^{-1} \circ \bar{r}$ is in $\Gamma(G)$ and satisfies $w \circ r=s$. Q.E.D.

4. The complexification as a group. In view of Proposition $3, G$ is a group precisely when $\omega * \nu$ exists in $\bar{W}$ for all $\omega, \nu \in G_{C}$. Theorem 1 implies that this is the case when $\omega * \nu$ exists in $\bar{W}$ for all $\omega, \nu \in G_{C}^{+}$. Thus one is led to seek a dense subspace of $H(T)$ common to the domains of all the operators $T_{\omega}, \omega \in G_{C}^{+}$. Since each $\omega \in G_{C}^{+}$is of the form $\exp (-i \alpha)$ for $\alpha \in \Lambda$, the problem is to find a $G$-invariant subspace $H(T)_{\varepsilon}$ of $H(T)$ such that, for each $\alpha \in \Lambda$,

$$
\sum_{n=0}^{\infty}\left(\frac{-i}{n !}\right)^{n} \alpha^{n} v \text { converges to an element of } H(T)_{\varepsilon} \quad\left(\forall v \in H(T)_{\varepsilon}\right) .
$$

Nelson's theorem [26, 4.4.5.7] on the density of analytic vectors for continuous Banach $G$-modules combined with (30) can be employed to find a $G$-invariant dense subspace $H(T)_{\omega}$ of $H(T)$ such that (33) holds for each, but only for each $\alpha$ in a neighborhood of 0 in $\Lambda$ (where $\Lambda$ bears its finest locally convex topology). This gives at best a local group structure. At this time, we can give only a partial solution to the problem.

We shall call $G$ entire if there exists a subspace $L_{\varepsilon}^{1}$ of $L^{1}(G)$ such that

$$
\sum_{n=0}^{\infty} \frac{1}{n !} z^{n} D_{s}^{n} f \in L_{\varepsilon}^{1} \quad\left(\forall z \in C, s \in \Gamma, f \in L_{e}^{1}\right)
$$

where the convergence is absolute. (Here $L^{1}(G)$ is viewed as a left $G$-module with action $x \cdot f \mid G \ni y \rightarrow f\left(x^{-1} y\right)$.)

Proposition 5. If $G$ is a product of entire groups $N$ and $K$, then $G$ is entire. 
Proof. If $f \in L_{\varepsilon}^{1}(N)$ and $h \in L_{\varepsilon}^{1}(R)$, then $f \otimes h \mid N \times K \ni(x, y) \rightarrow f(x) h(y)$ is in $L^{1}(G)$. IF $s$ is in $\Gamma(G)$, then there exist $m \in \Gamma(N)$ and $r \in \Gamma(K)$ such that $s(t)=(m(t), r(t))$ and so

$$
D_{s}^{n}(f \otimes h)=\sum_{k=0}^{n}\left(\begin{array}{l}
n \\
k
\end{array}\right) D_{m}^{k} f \otimes D_{r}^{n-k} h
$$

Thus

$$
\sum_{n=0}^{\infty} \frac{1}{n !}\left\|z^{n} D_{s}^{n}(f \otimes h)\right\|_{1} \leqslant\left(\sum_{i=1}^{\infty} \frac{1}{i !}\left\|z^{i} D_{m}^{i} f\right\|_{1}\right)\left(\sum_{j=1}^{\infty} \frac{1}{j !}\left\|z^{j} D_{r}^{j} h\right\|_{1}\right)<\infty
$$

and

$$
\sum_{n=0}^{\infty} \frac{1}{n !} z^{n} D_{s}^{n}(f \otimes h)=\left(\sum_{i=1}^{\infty} \frac{1}{i !} z^{i} D_{m}^{i} f\right) \otimes\left(\sum_{j=1}^{\infty} \frac{1}{j !} z^{j} D_{r}^{j} h\right) .
$$

Hence we may let $L_{\varepsilon}^{1}(G)$ be the linear span of $\left\{f \otimes h: f \in L_{\varepsilon}^{1}(N), h \in L_{\varepsilon}^{1}(R)\right\}$. Q.E.D.

COROllary 1. If $G$ is a SIN group (i.e. $G$ possesses a basis of neighborhoods invariant under inner automorphisms), then $G$ is entire. In particular, Abelian and compact groups are entire.

Proof. Connected SIN groups are products of compact groups and copies of $\mathbf{R}$. If $G$ is compact, we may take the space of trigonometric polynomials for $L_{\varepsilon}^{1}$ (see [18]). If $G=\mathbf{R}$, we may take the linear span of all functions

$$
\mathbf{R} \ni t \rightarrow \exp \left(-b(t-a)^{2}\right) \quad \text { where } b>0 \text { and } a \in \mathbf{R} \text {. Q.E.D. }
$$

Proposition 6. If $N$ is a closed normal subgroup of an entire group $G$, then $G / N$ is entire.

Proof. Let $p \mid G \rightarrow G / N$ be the canonical quotient epimorphism. Equip $N$ and $G / N$ with left Haar measure $\nu$ and $\theta$, respectively, such that, if

$$
f^{0} \mid G / N \ni p(x) \rightarrow \int_{N} f(x y) d \nu(y) \quad\left(\forall f \in L^{1}(G)\right),
$$

then

$$
\int_{G / N} f^{0} d \theta=\int_{G} f d \lambda
$$

and $L^{1}(G) \mid f \rightarrow f^{0}$ is an epimorphism onto $L^{1}(G / N)[12,28.54]$. Let $s \in \Gamma$ and $f \in L_{\varepsilon}^{1}(G)$ be arbitrary. Then, for each $h \in L^{\infty}(G / N)$,

$$
\begin{aligned}
\left|\int_{G / N}\left(\frac{p \circ s(t) \cdot f^{0}-f^{0}}{t}-\left(D^{s} f\right)^{0}\right) \cdot h d \theta\right| & =\left|\int_{G}\left(\frac{s(t) \cdot f-f}{t}-D^{s f}\right) \cdot h \circ p d \lambda\right| \\
& \leqslant\left\|\frac{s(t) \cdot f-f}{t}-D^{s} f\right\|_{1} \cdot\|h\|_{\infty}
\end{aligned}
$$

which implies that $f^{0} \in L^{1}(G / N)_{\infty}$ and $D_{p \circ s} f^{0}=\left(D_{s} f\right)^{0}$. 
For $z \in \mathbf{C}$, we have by (35a)

$$
\sum_{n=0}^{\infty} \frac{1}{n !}\left\|z^{n} D_{p \circ s}^{n} f^{0}\right\|_{1}=\sum_{n=0}^{\infty} \frac{1}{n !}\left\|z^{n} D_{s}^{n} f\right\|_{1}<\infty
$$

and

$$
\sum_{n=0}^{\infty} \frac{1}{n !} z^{n} D_{p \circ s}^{n} f^{0}=\left(\sum_{n=0}^{\infty} \frac{1}{n !} z^{n} D_{s}^{n} f\right)^{0} .
$$

Thus if we define $L_{\varepsilon}^{1}(G / N)$ to be $\left\{f^{0}: f \in L_{\varepsilon}^{1}(G)\right\}$, it follows from Theorem 2 that (34) holds. That $L_{\varepsilon}^{1}(G / N)$ is dense in $L^{1}(G / N)$ follows from the fact that the map $L^{1}(G) \ni f \rightarrow f^{0} \in L^{1}(G / N)$ is a continuous epimorphism. Q.E.D.

A partial converse to Proposition 6 holds in that, if $G / N$ is entire, then evidently (36) (34) holds when $L_{\varepsilon}^{1} \equiv\left\{f \circ p: f \in L_{\varepsilon}^{1}(G / N)\right\}$.

Boseck [4] and Lashof [15] showed that many statements hold for $G$ precisely when they hold for all the groups $G / N$ of (31). Proposition 6 and (36) imply that

(37) $G$ is entire iff $G / N$ is entire $(\forall N \in \mathcal{G})$ where $G \cong \operatorname{proj} \lim _{N \in \mathcal{G}} G / N$ is in (31).

Proposition 7. If $G$ is entire, then $G_{\mathbf{C}}$ is a group.

Proof. The set $M \equiv\left\{T_{f} v: f \in L_{\varepsilon}^{1}, v \in H(T)\right\}$ is a dense subset of $H(T)$. For each $\alpha \in \Lambda, f \in L_{\varepsilon}^{1}, v \in H$, and $z \in \mathrm{C}$ we have (where $s=s^{\alpha}$ )

$$
\sum_{n=0}^{\infty}\left|\frac{z^{n}}{n !} T_{\alpha}^{n}\left(T_{f} v\right)\right|=\sum_{n=0}^{\infty}\left|\frac{z^{n}}{n !} T_{D_{s}^{n} f} v\right| \leqslant \sum_{n=0}^{\infty}\left\|\frac{z^{n}}{n !} D_{s}^{n} f\right\|_{1}|v|<\infty
$$

so $T_{f} v$ is the domain of $T_{\text {exp }}(z \alpha)$. Further,

$$
T_{\exp (z \alpha)}\left(T_{f} v\right)=T_{\sum_{n-1}^{\infty}\left(z^{n} / n !\right) D_{s}^{n f}}(v),
$$

so by Proposition $1, M$ is $G_{\mathrm{C}}$-invariant. Hence $T_{\beta} \circ T_{\omega}$ is well defined for each $\beta, \omega \in G_{\mathbf{C}}$; thus Proposition 3 implies that $G_{\mathbf{C}}$ is a group. Q.E.D.

5. The complexification and the regular representation. If $V$ is any faithful continuous unitary representation of $G$, and $S$ is the canonical "extension" to $\bar{W}(G)$, then

(38) $S \mid G_{\mathbf{C}}$ is faithful.

To see this note, that by Theorem 1, (38) holds if $S$ is faithful on $G_{\mathrm{C}}^{+}$which, by Proposition 2, holds when $S$ is faithful on $\Lambda$. Since $V_{s^{\alpha}(t)}=S_{\exp (t \alpha)}$ for all $\alpha \in \Lambda$ and $t \in \mathbf{R}$, it follows from Proposition 4 and the faithfulness of $V$ that $S$ is faithful on $\Lambda$.

In particular, $G_{\mathbf{C}}$ is faithfully represented on $L^{2}(G)$ by the left regular representation $R$ of $W$. It is possible to base the theory of $G_{\mathrm{C}}$ on $R(W)$ rather than $W$, rewriting (20) as

$$
T_{\omega}^{f} \otimes T_{\omega}^{h}=T_{\omega}^{f, h} \quad(\forall f, h \in P \cap A(G))
$$

where $A(G)$ is the Fourier algebra. The only major change would be use of the Eymard duality theorem [10, 3.34] instead of Walter's theorem. 
Another faithful representation of $G$, the one to which the sequel is directed, is the product of all continuous, irreducible, unitary, representations, one from each unitary equivalence class.

6. The dual of $G$. Since (irreducible representation) duality theory has only been successfully developed for "tame" groups, throughout the sequel $G$ will be assumed to be a separable, Type I, connected, locally compact group.

Recall that a $W^{*}$-representation $S$ of $W$ is primary (or factorial) if $S(W) \cap$ $S(W)^{\prime}=\mathrm{C} I$. To say that a separable group $G$ is of Type $\mathrm{I}$, is equivalent to each of the following statements $[8,9.1]$ :

(39a) each primary $W^{*}$-representation of $W$ is a multiple of an irreducible representation;

(39b) irreducible $W^{*}$-representations of $W$ with common kernel are unitarily equivalent;

(39c) if $S$ is an irreducible $W^{*}$-representation of $W$, then $S(C)$ contains all compact operators.

Let $\Sigma=\Sigma(G)$ be the family of minimal central projections in $W^{+}$. If $\pi$ is the identity of the kernel of an irreducible $W^{*}$-representation of $W$, then $(\iota-\pi) * W$ is isomorphic to the algebra of all continuous linear operators on the representation space, so $\iota-\pi$ is in $\Sigma$. Conversely, if $\tau$ is in $\Sigma$, and $S$ is a faithful $W^{*}$-representation of $\tau * W$, then $S$ is primary so (39a) implies that $\iota-\tau$ is the identity of the kernel of an irreducible representation. Hence

(40) $\{\pi * W: \iota-\pi \in \Sigma\}$ is the set of primitive ideals of $W$ (a primitive ideal being the kernel of an irreducible representation).

The dual of a locally compact group is "classically" considered to be the family of unitary equivalence classes of continuous, irreducible, unitary representations of $G$; or, what amounts to the same thing, the family of unitary equivalence classes of irreducible $W^{*}$-representations of $W$. This being a rather cumbersome structure, we adduce (39b) and (40) to justify calling $\Sigma$ the dual of $G$.

The family 9 of closed two-sided ideals in $C(G)$ can be employed to describe the Jacobson topology on $\Sigma$ :

$$
\{\pi \in \Sigma: \pi * a \neq 0(\exists a \in J)\} \quad(J \in \mathscr{Y})
$$

is a listing of the open sets. The $\sigma$-algebra $\mathfrak{B}$ of Borel sets generated by the Jacobson topology makes $\Sigma$ a standard Borel space (i.e., isomorphic to $[0,1]$ as a Borel or measurable space) $[8,4 . b .1]$. We write $\mathfrak{M}(\Sigma)$ for the set of all $\sigma$-finite Borel measures on $\Sigma$.

Let $X=X(G)$ be the family of extreme points of the convex set $\{f \in P$ : $f(e) \leqslant 1$. When $G$ is Abelian, $X$ is the character group of $G$; in the general case $X$ is the set of repsresentative functions of the continuous irreducible representations of $G$. Thus, to each $f \in X$ corresponds a unique element $\pi_{f} \in \Sigma$ such that

(42) $\left.T^{f}\right|_{\pi_{f}, W}$ is an isomorphism onto $\mathcal{L}(H(f))$

(where $\mathcal{L}(H(f))$ is the von Neumann algebra of all bounded linear operators on $H(f))$. For $\pi \equiv \pi_{f}$ and $\omega \in \pi * W^{+}$, we define

$$
\operatorname{tr}_{\pi}(\omega) \equiv \operatorname{Tr}\left(T_{\omega}^{f}\right)
$$


where $\operatorname{Tr}$ is the trace on $\mathcal{L}(H(f))^{+}$. The function $\operatorname{tr}_{\pi}$ is independent of the $f \in X$ (satisfying $\pi=\pi_{f}$ ) employed in its construction. The Banach algebra of trace-class operators in $\mathcal{L}(H(f))$ transfers to $\pi * W$ :

$$
W_{\pi, 1} \equiv\left\{\omega \in \pi * W:\|\omega\|_{\pi, 1} \equiv \operatorname{tr}_{\pi}\left(\omega^{\sim} * \omega\right)^{1 / 2}<\infty\right\}
$$

and $\operatorname{tr}_{\pi}$ extends uniquely to a linear functional on $W_{\pi, 1}$.

We define the set $\operatorname{tm}(\Sigma)$ of temperate measurable operator fields on $\Sigma$ to be the set of all $\omega \in \Pi_{\pi \in \Sigma} W_{\pi, 1}$ such that the function $\Sigma \ni \pi \rightarrow \operatorname{tr}_{\pi}\left(\beta * \omega_{\pi}\right)$ is Borel measurable for each $\beta \in C$. Denote by $m(\Sigma)$ the set of all $\theta \in \Pi_{\pi \in \Sigma} \pi * W$ such that $\Sigma \ni \pi \rightarrow \operatorname{tr}_{\pi}\left(\theta_{\pi} * \omega_{\pi}\right)$ is Borel measurable for each $\omega \in \operatorname{tm}(\Sigma)$, and let $m^{\infty}(\Sigma)$ be the set of all $\omega \in m(\Sigma)$ such that

$$
\|\omega\|_{\infty} \equiv \sup \left\{\left\|\omega_{\pi}\right\|_{\boldsymbol{w}}: \pi \in \Sigma\right\}<\infty .
$$

Then $m^{\infty}(\Sigma)$ is evidently a $C^{*}$-algebra.

The Fourier-Stieltjes transformation ${ }^{\wedge} \mid W \rightarrow \Pi_{\pi \in \Sigma} \pi * W$ is defined by

$$
\hat{\omega}(\pi) \equiv \omega * \pi \quad(\forall \omega \in W, \pi \in \Sigma) .
$$

PROPOSITION 8. The Fourier-Stieltjes transformation is a $C^{*}$-algebra homomorphism of $W$ onto $m^{\infty}(\Sigma)$. If $z \equiv \sup _{\pi \in \Sigma} \pi$, then the restriction of ' to $z * W$ is an isomorphism onto $m^{\infty}(\Sigma)$.

Proof. Let $\omega \in W$ and $\theta \in \operatorname{tm}(\Sigma)$ be arbitrary. Then $\omega$ is the $\sigma\left(W, W_{*}\right)$-limit of a sequence $\omega_{n}$ in $C$. Since each of the functions $\Sigma \ni \pi \rightarrow \operatorname{tr}_{\pi}\left(\omega_{n} * \theta_{\pi}\right)$ is Borel measurable, so is $\Sigma \ni \pi \rightarrow \operatorname{tr}_{\pi}\left(\omega * \theta_{\pi}\right)$. Hence $\hat{\omega} \in m^{\infty}(\Sigma)$. That ${ }^{\wedge}$ is a ${ }^{*}$-homomorphism is trivial, as is the fact that ${ }^{\wedge} \mid z * W \rightarrow m^{\infty}(\Sigma)$ is a monomorphism.

Let $\theta \in m^{\infty}(\Sigma)$ be arbitrary. It is a consequence of $[8,8.4 .1]$ that, for each $\mu \in \mathfrak{M}(\Sigma)$,

$$
A(\mu) \equiv\left\{\chi \in W:\|\chi\|_{W} \leqslant\|\theta\|_{\infty}, \hat{\chi}=\theta \mu \text {-a.e. }\right\}
$$

is nonvoid. The partial ordering $\ll$ on $\mathfrak{M}(\Sigma)$ induced by "absolute continuity" is a direction. The net $\{A(\mu)\}_{\mu \in \mathfrak{M}(\Sigma)}$ consists of $\sigma\left(W, W_{*}\right)$-compact, nonvoid subsets of $W$ satisfying $A(\mu) \subset A(\nu)$ whenever $\nu \ll \mu$. Thus $\cap_{\mu \in \mathfrak{N}(\Sigma)} A(\mu)$ contains an element $\omega$. Evidently $\hat{\omega}=\theta$, whence follows that $(z * \omega)^{\hat{\alpha}}=\theta$, which proves that $\hat{\imath} \mid z * W \rightarrow m^{\infty}(\Sigma)$ is surjective. Q.E.D.

7. Bochner's theorem. One of the most useful tools in exploiting the FourierStieltjes transform on Abelian groups is Bochner's theorem [12, 33.3], which identifies $B(G)$ with the measure algebra of the dual group. Our next goal, attained in Theorem 3 below, is to establish such a theorem for a tame group. The first step is an improvement of a result of $\mathrm{J}$. Tomiyama $[8,4.2 .5]$.

Proposition 9. Let $A$ be a Type I $C^{*}$-algebra and $\left\{V^{j}\right\}_{j \in n}$ a finite set of pairwise inequivalent nonzero irreducible ${ }^{*}$-representations of $A$ on Hilbert space. For each representation $V^{j}$, let $T_{j}$ be a compact operator on the representation space $\mathfrak{G}_{j}$ of $V^{j}$. Then there exists $f \in A$ such that $V_{f}^{j}=T_{j}$ for all $j \in \underline{n}$.

Proof. Proposition 9.1 holds for $n=1$ by Sakai's theorem [8, 9.5.9]. Suppose that it has been established for $n-1$, for all Type I $C^{*}$-algebras. 
Claim 1. There is a permutation $\theta$ of $\underline{n} \equiv\{1,2, \ldots, n\}$ such that $\operatorname{Ker} V^{\theta(j)} \not$ $\operatorname{Ker} V^{\theta(n)}$ for all $j \in n-1$. Suppose $k \in \bar{n}$ is maximal with the property that there exists an injection $\tau \mid \underline{\underline{k} \rightarrow \underline{n}}$ satisfying $\operatorname{Ker} V^{\tau(j)} \not \operatorname{Ker} V^{\tau(k)}$ for all $j \in k-1$, and

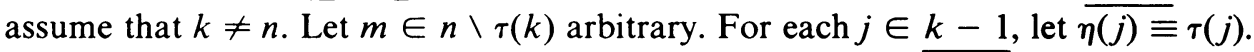
If $\operatorname{Ker} V^{m} \not \subset \operatorname{Ker} V^{\tau(k)}$, let $\eta(k) \equiv m$ and $\eta(k+1)=\tau(k)$, which would contradict the maximality of $k$. If $\operatorname{Ker} V^{m} \subset \operatorname{Ker} V^{\tau(k)}$, then clearly $\operatorname{Ker} V^{\tau(j)} \not \operatorname{Ker} V^{m}$ for all $j \in k-1$, and let $\eta(k) \equiv \tau(k)$ and $\eta(k+1) \equiv m$; since primitive ideals are distinct for Type I algebras [8, 4.3.7], we have $\operatorname{Ker} V^{\tau(k)} \not V^{m}$, which contradicts the maximality of $k$.

Let $\theta$ be as in the statement of Claim 1, and let $J \equiv\left\{f \in A: V_{f}^{\theta(n)}\right.$ is compact $\}$, which is a nonzero ideal of $A$ and thus Type $\mathrm{I}$.

Claim 2. For each $k \in n-1,\left.V^{\theta(k)}\right|_{J} \neq 0$. We may assume $n \geqslant 2$. Let $T$ be a compact nonzero projection in $\mathfrak{L}\left(H\left(V^{\theta(k)}\right)\right)$. By our inductive hypothesis, there exist $p, q \in A$ such that $V_{p}^{\theta(j)}=0$ for all $j \in n-1 /\{k\}, V_{p}^{\theta(k)}=T=V_{q}^{\theta(k)}$, and $V_{q}^{\theta(n)}$ $=0$. Clearly, $V_{p q}^{\theta(k)}=T \neq 0$ and $p q \in J$.

Claim 3. For $j, k \in n-1$ with $j \neq k,\left.V^{\theta(j)}\right|_{J}$ and $\left.V^{\theta(k)}\right|_{J}$ are inequivalent. We may assume $n>2$. Assume Claim 3 is false and let $U$ be the unitary operator such that $V_{g}^{\theta(j)}=U \circ V_{g}^{\theta(k)} \circ U^{-1}$ for all $g \in J$. By hypothesis there exists $h \in A$ such that $V_{h}^{\theta(j)} \neq U \circ V_{h}^{\theta(k)} \circ U^{-1}$. Hence $V_{h}^{\theta(j)} \circ K \neq U \circ V_{h} \circ U^{-1} \circ K$ for some compact operator $K$. By our inductive hypothesis, there exists $g \in A$ such that $V_{g}^{\theta(j)}=K$ and $V_{g}^{\theta(n)}=0$. Thus $h g$ is in $J$ and

$$
\begin{aligned}
V_{h g}^{\theta(j)} & =V_{h}^{\theta(j)} \circ K \neq U \circ V_{h}^{\theta(k)} \circ U^{-1} \circ V_{g}^{\theta(j)} \\
& =U \circ V_{h}^{\theta(k)} \circ U^{-1} \circ U \circ V_{g}^{\theta(k)} \circ U^{-1}=U \circ V_{h g}^{\theta(k)} \circ U^{-1},
\end{aligned}
$$

which is absurd.

It follows from Claim 2 and $[8,2.11 .2]$ that $\left.V^{\theta(k)}\right|_{J}$ is nonzero irreducible for each $k \in n-1$. Thus, our inductive hypothesis and Claim 3 yield some $h \in J$ such that $V_{h}^{\theta(k)}=T_{\theta(k)}$ for all $k \in n-1$. Let $L$ be the ideal $\cap_{k \in n-1} \operatorname{Ker} V^{\theta(k)}$. By Claim 1 and $[8,2.11 .4]$, we have $\overline{L \subset \mathrm{K}} \operatorname{er} V^{\theta(n)}$. Hence $\left.V^{\theta(n)}\right|_{L}$ is nonzero irreducible and so, since $L$ is Type $\mathrm{I}, V^{\theta(n)}(L)$ contains all compact operators on the representation space of $V^{\theta(n)}$. Choose $g \in L$ such that $V_{g}^{\theta(n)}=T_{\theta(n)}-V_{h}^{\theta(n)}$. Let $f \equiv g+h$. Q.E.D.

Each space $W_{\pi, 1}, \pi \in \Sigma$, has norm \|\|$_{\pi, 1} \mid W_{\pi, 1} \ni \omega \rightarrow \operatorname{tr}_{\pi}\left(\omega^{\sim} * \omega\right)^{1 / 2}$ which makes (47) $W_{\pi, 1}$ linearly isometric to the predual of $\pi * W$ where the isomorphism $E_{\pi} \mid W_{\pi, 1} \rightarrow W_{*}$ is given by

$$
\eta\left(E_{\pi}(\omega)\right)=\operatorname{tr}_{\pi}(\eta * \omega) \quad\left(\forall \eta \in W, \omega \in W_{\pi, 1}\right) .
$$

Let $\operatorname{tm}_{00}(\Sigma)$ be the set of all $\theta \in \operatorname{tm}(\Sigma)$ such that $\theta_{\pi} \neq 0$ only for a finite set of $\pi$, and let $\operatorname{tm}_{1}(\Sigma)$ be the set of all $\theta \in \operatorname{tm}(\Sigma)$ such that

$$
\|\theta\| \equiv \sum_{\pi \in \Sigma}\left\|\theta_{\pi}\right\|_{\pi, 1}<\infty
$$

Then $\operatorname{tm}_{1}(\Sigma)$ is a Banach *-algebra in which $\operatorname{tm}_{00}(\Sigma)$ is a dense ideal. For each $\theta \in \operatorname{tm}_{1}(\Sigma)$ and $\pi \in \Sigma$, (43) implies

$$
\left\|E_{\pi}\left(\theta_{\pi}\right)\right\|_{W_{*}} \leqslant\left\|\theta_{\pi}\right\|_{\pi, 1}
$$


so the series $\Sigma_{\pi \in \Sigma} E_{\pi}\left(\theta_{\pi}\right)$ converges in $W_{*}$. We define ${ }^{\sharp} \mid \mathrm{tm}_{1}(\Sigma) \rightarrow B(G)$ by appealing to (15) to find the unique $\theta^{\#} \in B$ such that

$$
F_{\theta^{\sharp}}=\sum_{\pi \in \Sigma} E_{\pi}\left(\theta_{\pi}\right)
$$

If $B$ is equipped with the norm \|\|$_{B}$ induced from $W_{*}$ by $F$, then it is evident from (49) and (50) that \# is norm nonincreasing.

Proposition 10. The map $\# \mid \operatorname{tm}_{1}(\Sigma) \rightarrow B(G)$ is a linear isometry onto a closed subspace of $B(G)$.

Proof. The only nontrivial thing to show is that \# is an isometry, and this will follow once we have established that the restriction of $\#$ to $\mathrm{tm}_{00}(\Sigma)$ is an isometry. Let $\theta \in \operatorname{tm}_{00}(\Sigma)$ be arbitrary and choose a finite subset $\Omega$ of $\Sigma$ such that $\pi \in \Omega$ whenever $\theta_{\pi} \neq 0$. For each $\pi \in \Sigma, T_{\theta_{\pi}}$ is a trace-class operator on $\pi(H(T))$ so there exists a compact operator $K_{\pi}$ on $\pi(H(T))$ of unit norm such that

$$
\operatorname{Tr}\left(K_{\pi} \circ T_{\theta_{\pi}}\right)=\left\|\theta_{\pi}\right\|_{\pi, 1} .
$$

Since $G$ is Type I, $C(G)$ is of Type I and it follows from (39) and Proposition 9 that there exists some $\omega \in C$ such that $T_{\pi \bullet \omega}=K_{\pi}$ for all $\pi \in \Omega$. Since each $K_{\pi}$ has norm 1 , we have

$$
\left\|\omega * \sum_{\pi \in \Omega} \pi\right\|_{W}=1
$$

From (51) and (50) it follows that

$$
\left\langle\omega * \sum_{\pi \in \Omega} \pi, \theta^{\sharp}\right\rangle=\sum_{\pi \in \Omega} \operatorname{tr}_{\pi}\left(\omega * \pi * \theta_{\pi}\right)=\sum_{\pi \in \Omega} \operatorname{Tr}\left(K_{\pi} \circ T_{\theta_{*}}\right)=\sum_{\pi \in \Omega}\left\|\theta_{\pi}\right\|_{\pi, 1} \cdot
$$

But (52) and (53) together imply that $\left\|\theta^{\sharp}\right\|_{B}>\|\theta\|$. Hence $\left\|\theta^{\sharp}\right\|_{B}=\|\theta\|$. Q.E.D.

Lemma 2. For $\pi \in \Sigma$ and $\theta \in W_{\pi, 1}^{+}$, let $f \equiv \theta^{\sharp}$. Then the representation $T^{f}$ is primary.

Proof. Since $\pi * W$ is simple, the representation $\left.T^{f}\right|_{\pi * W}$ is an isomorphism. Similarly, if $\tau$ is a minimal projection of $W_{\pi, 1}$ and $h=\tau^{\sharp}$, then $\left.T^{h}\right|_{\pi, W}$ is an isomorphism. Thus by [8, 5.3.1(ii)], $\left.T^{f}\right|_{\pi, W}$ and $\left.T^{h}\right|_{\pi, W}$ are quasi-equivalent. Since $T^{h}$ is irreducible, $T^{f}$ is primary. Q.E.D.

Lemma 3. Let $V$ be a $W^{*}$-representation of $W$ on a Hilbert space $H(V)$, and suppose that $\int_{\Sigma} V^{\pi} d \mu(\pi)$ is a disintegration of $V$ such that each $V^{\pi}(\pi \in \Sigma)$ is quasi-equivalent to the $W^{*}$-representation of $W$ associated with $\pi$. Then the disintegration is central (i.e. $V(W) \cap V(W)^{\prime}$ consists precisely of the diagonalizible operators).

Proof. Let $w \in W$ satisfy $V_{w} \in V(W)^{\prime}$. Then $V_{w}^{\pi}$ is in $V^{\pi}(W)^{\prime}$ for $\mu$-almost all $\pi \in \Sigma$. Since $V^{\pi}$ is primary for each $\pi \in \Sigma$, we have $V^{\pi}(W)^{\prime} \cap V^{\pi}(W)=\mathbf{C I}$; consequently $V_{w}$ is diagonalizible. 
Now let $T$ be an arbitrary (bounded) diagonalizible operator on $H(V)$, and let $\int_{\Sigma} T^{\pi} d \mu(\pi)$ be its decomposition. For each $\pi \in \Sigma$, let $H(\pi)_{r}$ be a subspace of the representation space $H(\pi)$ of $V^{\pi}$ such that the restriction of $V^{\pi}$ to $H(\pi)_{r}$ is irreducible.

The restriction $T^{r}$ of $T$ to $H(V)_{r} \equiv \int_{\Sigma} H(\pi)_{r} d \mu(\pi)$ is obviously diagonalizible. Since the restriction $V^{r}$ of $V$ to $H(V)_{r}$ is a direct integral of irreducible representations, it follows from [8, Théorème 8.6.5] that $T^{r}$ is of the form $V_{\eta}^{r}$ for some $\eta \in W$. Let $\pi \in \Sigma$ be arbitrary and write $V^{r, \pi}$ for the restriction of $V^{r}$ to $H(\pi)_{r}$. Then $V_{\eta}^{r, \pi}$ is a scalar multiple $k_{\pi}$ of the identity. Since $R_{\eta}$ is also $k_{\pi}$ times the identity for any representation $R$ of $W$ equivalent to $V^{r, \pi}$, it follows that $V_{\eta}^{\pi}$ must be $k_{\pi}$ times the identity. But $T^{\pi}$ being a scalar multiple of the identity whose restriction to $H(\pi)_{r}$ is $V_{\eta}^{r, \pi}$, it follows that $T^{\pi}=V_{\eta}^{\pi}$. Consequently, $T=V_{\eta}$. That $T$ is in $V(W)^{\prime}$ is trivial. Q.E.D.

By an operator-measure $\theta \mu$ we shall mean an equivalence class of ordered pairs $(\theta, \mu) \in \operatorname{tm}(\Sigma)^{+} \times \mathfrak{M}(\Sigma)^{+}$, equivalence being defined by

$$
\theta \mu \sim \eta \nu \Leftrightarrow \theta=\eta \cdot \frac{d \nu}{d \mu} \quad \mu \text {-a.e. }
$$

and $\mu$ and $\nu$ are equivalent measures. The set of all such $\theta \mu$ shall be written $\mathscr{D M}(\Sigma)$. For $\theta \mu \in \mathfrak{D M}(\Sigma)$, define

$$
\|\theta \mu\|_{\bigcirc \mathfrak{N}} \equiv \int_{\Sigma}\left\|\theta_{\pi}\right\|_{\pi, 1} d \mu(\pi)
$$

and write $\mathfrak{D M}_{\mathrm{bd}}(\Sigma)$ for the set of all $\theta \mu$ such that $\|\theta \mu\|_{\mathfrak{O M}}<\infty$. The transformation $\checkmark \mathfrak{D M}_{\text {bd }}(\Sigma) \rightarrow P(G)$ is defined by

$$
(\theta \mu)^{\sim} \mid G \ni x \rightarrow \int_{\Sigma} \operatorname{tr}_{\pi}\left(\gamma(x) * \theta_{\pi}\right) d \mu(\pi) \quad\left(\forall \theta \mu \in \mathfrak{D M}_{\mathrm{bd}}(\Sigma)\right),
$$

so that, as well,

$$
\left\langle\omega,(\theta \mu)^{-}\right\rangle=\int_{\Sigma} \operatorname{tr}_{\pi}\left(\omega * \theta_{\pi}\right) d \mu(\pi) \quad(\forall \omega \in W) .
$$

If $\theta \in \operatorname{tm}_{1}(\Sigma)^{+}$and $\kappa$ is counting measure on $\left\{\pi \in \Sigma: \theta_{\pi} \neq 0\right\}$, then

$$
\theta^{\#}=(\theta \kappa)^{-} \text {. }
$$

THEOREM 3. The map $\nmid \mid \mathfrak{M}_{\mathrm{bd}}(\Sigma) \rightarrow P(G)$ is a bijection and, for each $\theta \mu \in$ $\mathfrak{S M}_{\mathrm{bd}}(\Sigma)$,

(i) $\left\|(\theta \mu)^{\sim}\right\|_{B}=\|\theta \mu\|_{o m}$.

Proof. Let $f \in P$ be arbitrary. The representation $T^{f}$ is unitarily equivalent to a direct integral $\int_{\Sigma} S^{\pi} d \mu(\pi)$ where $\mu$ is a measure in $\mathfrak{M}(\Sigma)^{+}$and each $S^{\pi}$ is a primary representation quasi-equivalent to the irreducible $W^{*}$-representation of $W$ associated with $\pi$. For each $\pi \in \Sigma$, write $H(\pi)$ for the representation space of $S^{\pi}$. Let $w$ be the cyclic vector of $\int_{\Sigma} H(\pi) d \mu(\pi)$ corresponding to $v_{f} \in H(f)$ by the unitary equivalence. By modification of a $\mu$-negligible set, if necessary, we may assume, that each $w_{\pi}$ is cyclic in $H_{\pi}$. 
Consider $\pi \in \Sigma$. Since $G$ is separable and $S$ cyclic, $H(\pi)$ is the direct sum $\bigoplus_{n=1}^{\infty} H_{\pi, n}$ of irreducible subspaces. Let $\sum_{n=1}^{\infty} w_{\pi, n}$ be the corresponding decomposition of $w_{\pi}$. Then each operator

$$
w_{\pi, n} \otimes w_{\pi, n}^{*} \mid H_{\pi, n} \ni v \rightarrow\left\langle w_{\pi, n}, v\right\rangle_{H(\pi)} w_{\pi, n}
$$

is a positive operator of trace $\left|w_{\pi, n}\right|^{2}$, so there exists $\eta_{\pi, n} \in W_{\pi, 1}$ such that

$$
\left\|\eta_{\pi, n}\right\|_{\pi, 1}=\left|w_{\pi, n}\right|^{2}, \quad S_{\eta_{n, n}}^{\pi}=w_{\pi, n} \otimes w_{\pi, n}^{*} .
$$

Since

$$
\sum_{n=1}^{\infty}\left\|\eta_{\pi, n}\right\|_{\pi, 1}=\sum_{n=1}^{\infty}\left|w_{\pi, n}\right|^{2}=\left|w_{\pi}\right|^{2}<\infty
$$

the series $\sum_{n=1}^{\infty} \eta_{\pi, n}$ converges to an element $\eta_{\pi}$ of $W_{\pi, 1}$. We have now defined an element $\eta$ of $\operatorname{tm}(\Sigma)$ satisfying, for all $\omega \in W$,

$$
\begin{aligned}
\langle\omega, f\rangle & =\left\langle T_{\omega}^{f} v_{f}, v_{f}\right\rangle_{H(f)}=\int_{\Sigma}\left\langle S_{\omega}^{\pi} w_{\pi}, w_{\pi}\right\rangle d \mu(\pi) \\
& =\int_{\Sigma} \sum_{n=1}^{\infty} \operatorname{Tr}\left(S_{\omega}^{\pi} \circ\left(w_{\pi, n} \otimes w_{\pi, n}^{*}\right)\right) d \mu(\pi) \\
& =\int_{\Sigma} \sum_{n=1}^{\infty} \operatorname{tr}_{\pi}\left(\omega * \eta_{\pi, n}\right) d \mu(\pi)=\int_{\Sigma} \operatorname{tr}_{\pi}\left(\omega * \eta_{\pi}\right) d \mu(\pi)=\left\langle\omega,(\eta \mu)^{\swarrow}\right\rangle .
\end{aligned}
$$

Applying (61) when $\omega=\imath$ yields (i).

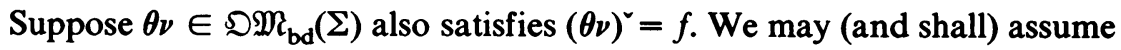
(62) $\mu=\nu$ if $\mu$ and $\nu$ are equivalent.

For each $\pi \in \Sigma$, write $f_{\pi}$ for $\left(\theta_{\pi}\right)^{\sharp}$. Let $V$ be the direct integral $\int_{\Sigma} T^{f_{*}} d \nu(\pi)$ and denote $\int_{\Sigma} H\left(f_{\pi}\right) d \nu(\pi)$ by $H(V)$. Since

$$
\begin{aligned}
\int_{\Sigma}\left|v_{f_{\pi}}\right|^{2} d \nu(\pi) & =\int_{\Sigma}\left\langle T_{\imath}^{f_{*}} v_{f_{\pi}}, v_{f_{*}}\right\rangle_{H\left(f_{*}\right)} d \nu(\pi) \\
& =\int_{\Sigma} \operatorname{tr}_{\pi}\left(\iota * \theta_{\pi}\right) d \nu(\pi)=\int_{\Sigma}\left\|\theta_{\pi}\right\|_{\pi, 1} d \nu(\pi)=\|\theta \nu\|_{\mathcal{R O}}<\infty,
\end{aligned}
$$

the vector $v \equiv \int_{\Sigma} v_{f_{z}} d \nu(\pi)$ exists. If $M$ is the orthogonal projection of $H(V)$ onto the minimal $V$-invariant subspace containing $v$, then $M=\int M^{\pi} d \nu(\pi)$ where $\nu$ -

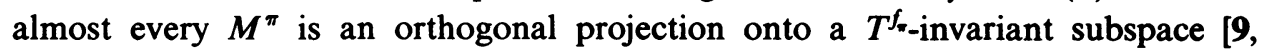
II.3.3, Théorème 3]; since $\int_{\Sigma} v_{f_{*}} d \nu(\pi)=v=M(v)=\int_{\Sigma} M^{\pi}\left(v_{f_{*}}\right) d \nu(\pi)$, it follows that $v_{f_{\pi}}=M^{\pi}\left(v_{f_{\pi}}\right) \nu$-almost everywhere and so, since each $v_{f_{\pi}}$ is cyclic in $H\left(f_{\pi}\right), M$ is the identity operator. Thus

(63) $v$ is $V$-cyclic for $H(V)$.

Furthermore, for each $\omega \in W$,

$$
\begin{aligned}
\left\langle V_{\omega} v, v\right\rangle_{H(V)} & =\int_{\Sigma}\left\langle T_{\omega}^{f_{*}} v_{f_{*}}, v_{f_{*}}\right\rangle_{H\left(f_{*}\right)} d v(\pi) \\
& =\int_{\Sigma} \operatorname{tr}_{\pi}\left(\omega * \theta_{\pi}\right) d \nu(\pi)=(\theta v)^{-}(\omega)=\langle\omega, f\rangle .
\end{aligned}
$$


It follows from (63) and (64) that $V$ is unitarily equivalent to $T^{f}$, and so to $\int_{\Sigma} S^{\pi} d \mu(\pi)$ as well. Lemma 2 implies that each representation $T^{f_{*}}, \pi \in \Sigma$, is primary. From Lemma 3 follows that the diagonal algebras of the given disintegrations of $T^{f}$ and $V$ are $T^{f}(W) \cap T^{f}(W)^{\prime}$ and $V(W) \cap V(W)^{\prime}$, respectively. Thus, if $U \mid H(V) \rightarrow H(f)$ maps $v$ to $w$ and implements the equivalence between $V$ and $T^{f}$, $U$ transforms the diagonal algebra of $\int_{\Sigma} V^{\pi} d \nu(\pi)$ into that of $\int_{\Sigma} S^{\pi} d \mu(\pi)$. It follows from (62) and [24, Proposition 8.27] that $\mu=\nu$ and there exist a Borel subset $\Omega$ of $\Sigma$ with $\nu$-negligible complement and a measurable field of unitary operators $U^{\pi} \mid H\left(f_{\pi}\right) \rightarrow H(\pi)$ such that

$$
U^{\pi} \circ S_{\omega}^{\pi} \circ\left(U^{\pi}\right)^{-1}=V_{\omega}^{\pi} \quad(\forall \pi \in \Omega, \omega \in W)
$$

and

$$
U=\int_{\Omega} U^{\pi} d \nu(\pi) .
$$

Since $U(v)=w$, it follows from (66) and (65) that $U^{\pi}\left(v_{f_{n}}\right)=w_{\pi}$ for all $\pi \in \Omega$. Thus, for each $\pi \in \Omega$ and $\omega \in W$,

$$
\begin{aligned}
\operatorname{Tr}\left(S_{\omega}^{\pi} \circ S_{\eta}^{\pi}\right) & =\operatorname{Tr}\left(S_{\omega}^{\pi} \circ \sum_{n=1}^{\infty} w_{\pi, n} \otimes w_{\pi, n}^{*}\right)=\left\langle S_{\omega}^{\pi} w_{\pi}, w_{\pi}\right\rangle_{f} \\
& =\left\langle\left(U^{\pi}\right)^{-1} \circ V_{\omega}^{\pi} \circ U^{\pi}\left(w_{\pi}\right), w_{\pi}\right\rangle_{f}=\left\langle V_{\omega}^{\pi}\left(v_{\pi}\right), v_{\pi}\right\rangle \\
& =\operatorname{Tr}\left(V_{\omega}^{\pi} \circ v_{\pi} \otimes v_{\pi}^{*}\right)=\operatorname{tr}_{\pi}\left(\omega * \theta_{\pi}\right) .
\end{aligned}
$$

It follows that $\eta=\theta \boldsymbol{\nu}$-almost everywhere on $\Sigma$. Q.E.D.

For each $\pi \in \Sigma$, let $\mathcal{T}_{\pi}(G)$ be the set $\left\{\theta^{\sharp:}: \theta \in W_{1, \pi}\right\}$ or, in other words, the closure in $B$ of the linear span of representative functions emanating from the irreducible representation of $G$ associated with $\pi$.

Corollary 2. Let $f \in P(G)$ be arbitrary. Then there exist an element $h$ of the Cartesian product $\Pi_{\pi \in \Sigma} \mathcal{T}_{\pi}(G)$ and a measure $\mu \in \mathfrak{M}(\Sigma)^{+}$such that

(i) $\langle\omega, f\rangle=\int_{\Sigma}\left\langle\omega, h_{\pi}\right\rangle d \mu(\pi)$, and

(ii) $\|f\|_{B}=\int_{\Sigma}\left\|h_{\pi}\right\|_{B} d \mu(\pi)$.

The pair $(h, \mu)$ satisfying (i) is unique ( $h$ being determined up to a $\mu$-negligible set) provided

(iii) $\left\|h_{\pi}\right\|_{B}=1$ for $\mu$-almost all $\pi \in \Sigma$.

Proof. By Theorem 3, $f=(\theta \mu)^{\swarrow}$ for some $\theta \mu \in \mathcal{S M}_{\text {bd }}(\Sigma)$. Letting $h_{\pi} \equiv \theta_{\pi}^{\sharp}$ for all $\pi \in \Sigma$, we have for all $\omega \in W$,

$$
\begin{aligned}
\langle\omega, f\rangle & =\left\langle\omega,(\theta \mu)^{\sim}\right\rangle=\int_{\Sigma} \operatorname{tr}_{\pi}\left(\omega * \theta_{\pi}\right) d \mu(\pi) \\
& =\int_{\Sigma}\left\langle\omega, \theta_{\pi}^{\sharp}\right\rangle d \mu(\pi)=\int_{\Sigma}\left\langle\omega, h_{\pi}\right\rangle d \mu(\pi)
\end{aligned}
$$

which proves (i). If $\omega=\iota$ in (i), we obtain (ii). The uniqueness of $(h, \mu)$ subject to (iii) follows from Theorem 3 and (54). Q.E.D. 
8. The complexification and the dual. The Fourier-Stieltjes transformation $\mid W \rightarrow$ $m^{\infty}(\Sigma)$ has a canonical extension. For each $\omega \in \bar{W}$, we define

$$
\left.\hat{\omega}_{\pi} \equiv \omega\right|_{\pi}, W_{*} \cap \operatorname{dom}(\omega)
$$

then

$$
(\overline{\pi * W})=\left\{\hat{\omega}_{\pi}: \omega \in \bar{W}\right\} \quad(\forall \pi \in \Sigma) .
$$

We write $\bar{m}(\Sigma)$ for the set of all $\hat{\omega}, \omega \in \bar{W}$. Evidently

$$
\bar{m}(\Sigma) \cong \overline{m^{\infty}(\Sigma)} \text {. }
$$

It follows from (38) and the fact that $G \ni x \rightarrow \gamma(x)^{\wedge}$ is faithful, that ${ }^{\wedge}$ is also faithful on $G_{\mathrm{C}}$ and $\Lambda$ : we write $G_{\mathrm{C}}^{\wedge}$ and $\Lambda^{\wedge}$ for the respective images by ${ }^{\wedge}$.

It is the business of the sequel to determine how $G_{\mathbf{C}}$ may be identified intrinsically in terms of $\bar{m}(\Sigma)$. Theorem 1 implies that it suffices to identify $G_{\gamma}^{\wedge}$ and $G_{\mathrm{C}}^{+\wedge}$, and it follows from Proposition 2 that, to find $G_{\mathrm{C}}^{+\wedge}$, it suffices to find $\Lambda^{\wedge}$, so our task will be done once we have characterized $G_{\gamma}^{\wedge}$ and $\Lambda^{\wedge}$.

In our terminology, Tatsuuma's duality theorem [13, Satz 11.4.2] can be stated as follows. An element $\zeta \in m^{\infty}(\Sigma)$ is in $G_{\gamma}^{\hat{\gamma}}$ provided each of the following three conditions is satisfied:

(70a) $\zeta_{\pi}$ is unitary for each $\pi \in \Sigma$;

if $R$ and $S$ are irreducible $W^{*}$-representations and $V$ is the $W^{*}$-representation of $W$ such that $(R \circ \gamma) \otimes(S \circ \gamma)=V \circ \gamma$, then, where $\eta$ is the element of $z * W$ satisfying $\hat{\eta}=\zeta$,

(70b) $R_{\eta} \otimes S_{\eta}=V_{\eta}$

if $\int_{\Sigma} L^{\pi} d \mu(\pi)$ is a direct integral of the left regular representation $L$ with each $L^{\pi}(W)$ quasi-equivalent to $\pi * W$, then

(70c) $\int_{\Sigma} L_{\eta_{\pi}}^{\pi} d \mu(\pi) \neq 0$ if $\eta \neq 0$.

Some such condition as (70c) is necessary to take into the account the "topological" character of $\Sigma$ (when $G$ is Abelian, $\Sigma$ is "replaced" by $X$ and (70c) is replaced by the condition that $\zeta$ be continuous). The condition (70c) however seems extraneous for a characterization of $G_{\gamma}^{\wedge}$ intrinsically in terms of $\Sigma$ and $m^{\infty}(\Sigma)$.

Our solution to the problem will be described in terms of quasi-multipliers. Let $Q M(C)$ be the set of all $\omega \in W$ such that $\alpha * \omega * \beta \in C$ whenever $\alpha, \beta \in C$, and let $q m^{\infty}(\Sigma)$ be the set of all $\theta \in m^{\infty}(\Sigma)$ such that $\zeta * \theta * \eta \in C^{\wedge}$ for all $\zeta, \eta \in C^{\wedge}$.

It has been shown [1, Theorem 4.1] that, for each $\omega \in Q M(C)$ and net $f_{\alpha}$ in $P$ satisfying $\sigma\left(W_{*}, C\right)-\lim _{\alpha} F_{f_{\alpha}}=F_{f}$ for $f \in P$,

$$
\lim _{\alpha}\left\langle\omega, f_{\alpha}\right\rangle=\langle\omega, f\rangle \text { if } \lim _{\alpha}\left\|f_{\alpha}\right\|=\|f\| .
$$

It is known [1, Corollary 3] that if $\Psi$ is any $W^{*}$-homomorphism of $W$, then

$$
\Psi(Q M(C))=\{\omega \in \Psi(W): \alpha * \omega * \beta \in \Psi(C)(\forall \alpha, \beta \in \Psi(C))\} .
$$

In particular,

$$
q m^{\infty}(\Sigma)=\{\hat{\omega}: \omega \in Q M(C)\} .
$$


Evidently, (73) implies

$$
G_{\gamma}^{\wedge} \subset q m^{\infty}(\Sigma) .
$$

For $\zeta \in m^{\infty}(\Sigma)$ and $f \in B(G)$ we define

$$
\langle\zeta, f\rangle_{\Sigma} \equiv\langle\eta, f\rangle
$$

where $\zeta=\hat{\eta}$ and $\eta \in z * W$.

THEOREM 4. A unitary element $\zeta$ of $q m^{\infty}(\Sigma)$ is in $G_{\gamma}^{\wedge}$ if and only if

$$
\langle\zeta, f\rangle_{\Sigma}\langle\zeta, h\rangle_{\Sigma}=\int_{\Sigma} \operatorname{tr}_{\pi}\left(\zeta_{\pi} * \theta_{\pi}\right) d \mu(\pi) \quad(\forall f, h \in X)
$$

where, for each pair $f, h \in X, \theta \mu$ is the unique element of $\mathscr{O M}(\Sigma)$ such that $(\theta \mu)^{\nu}=f h$.

Proof. It follows from (73) that $\zeta=\hat{\eta}$ for some $\eta \in Q M(C)$. That (i) holds means

$$
\langle\eta, f\rangle\langle\eta, h\rangle=\langle\eta, f h\rangle \quad(\forall f, h \in X) .
$$

If $A$ is the convex cone generated by $X$ in $P$, it follows from (76) that

$$
\langle\eta, f\rangle\langle\eta, h\rangle=\langle\eta, f h\rangle \quad(\forall f, h \in A) .
$$

If $f$ and $h$ in $P$ are arbitrary, it follows from the Krein-Milman theorem that there exist nets $f_{\alpha}$ and $h_{\beta}$ in $A$ such that

$$
\left\|f_{\alpha}\right\|_{B} \rightarrow\|f\|_{B}, \quad\left\|h_{\beta}\right\|_{B} \rightarrow\|h\|_{B}
$$

and

$$
f_{\alpha} \rightarrow f, \quad h_{\beta} \rightarrow h \quad \text { in the topology } \sigma\left(W_{*}, C\right) .
$$

It follows from (78a), (78b) and Gelfand's theorem [12, 32.40] that $f_{\alpha} \rightarrow f$ and $h_{\beta} \rightarrow h$ in the compact-open topology.

Thus $f_{\alpha} h_{\beta} \rightarrow f h$ in the compact open topology so, by Gelfand's theorem again,

$$
f_{\alpha} h_{\beta} \rightarrow f h \text { in the topology } \sigma\left(W_{*}, C\right) .
$$

It now follows from (71) and (77) that

$$
\langle\eta, f\rangle\langle\eta, h\rangle=\lim _{\alpha, \beta}\left\langle\eta, f_{\alpha}\right\rangle\left\langle\eta, h_{\beta}\right\rangle=\lim _{\alpha, \beta}\left\langle\eta, f_{\alpha} h_{\beta}\right\rangle=\langle\eta, f h\rangle .
$$

From (80) follows

$$
\langle\eta, f\rangle\langle\eta, h\rangle=\langle\eta, f h\rangle .
$$

Walter's duality theorem and (81) imply that $\eta=\gamma(x)$ for some $x \in G$. Hence $\zeta=\gamma(x)$. Q.E.D.

The quasi-strict topology on $q m^{\infty}(\Sigma)$ is that induced by the seminorms

$$
q m^{\infty}(\Sigma) \ni \theta \rightarrow\|\zeta * \theta * \eta\|_{\infty} \quad\left(\zeta, \eta \in C^{\wedge}\right)
$$

When $G$ is Abelian, $q m^{\infty}(\Sigma)$ may be identified with the bounded, continuous, complex-valued functions on the dual group $X$, and, on \|\|$_{\infty}$-bounded subsets of $q m^{\infty}(\Sigma)$, the quasi-strict topology and the compact-open topology agree under this identification. Since the Fourier-Stieltjes transformation " is an isometry when 
restricted to $C[8,2.7 .3]$, it follows from (73) that, for $\omega \in Q M(C)$ and any net $\omega_{\alpha}$ in $Q M(C)$,

$$
\hat{\omega_{\alpha}} \rightarrow \hat{\omega} \quad \text { quasi-strictly } \Leftrightarrow\left\|\varepsilon *\left(\omega_{\alpha}-\omega\right) * \kappa\right\|_{W} \rightarrow 0 \quad(\forall \varepsilon, \kappa \in C) .
$$

Proposition 11. The map $\hat{\gamma} \mid G \ni x \rightarrow \gamma(x)^{\wedge} \in q m^{\infty}(\Sigma)$ is a homeomorphism when $q m^{\infty}(\Sigma)$ bears the quasi-strict topology.

Proof. That $\hat{\gamma}$ is continuous is a consequence of [11, 20.4]. If $\gamma\left(x_{\alpha}\right)^{\wedge}$ converges to $\gamma(x)^{\wedge}$ in the quasi-strict topology, then (83) and [17, Theorem 7] imply

$$
\lim _{\alpha, \beta}\left\langle\gamma\left(x_{\alpha}\right), f\right\rangle=\langle\gamma(x), f\rangle \quad(\forall f \in B) .
$$

That $x_{\alpha}$ converges to $x$ now follows from Walter's theorem [25, Theorem 1]. Q.E.D.

THEOREM 5. An element $\beta$ of $\bar{m}(\Sigma)$ is in $\Lambda^{\wedge}$ if and only if all the following conditions are satisfied:

(i) $-\beta_{\pi}=\beta_{\pi}^{\sim}(\forall \pi \in \Sigma)$;

(ii) $\langle\exp (t \beta), f\rangle_{\Sigma}\langle\exp (t \beta), h\rangle_{\Sigma}=\int_{\Sigma} \operatorname{tr}_{\pi}\left(\theta_{\pi} * \exp \left(t \beta_{\pi}\right)\right) d \mu(\pi)$ for all $t \in R$ and $f, h \in X$ (where $\theta \mu \in D_{\mathbb{M}_{\mathrm{bd}}}(\Sigma)$ satisfies $\left.(\theta \mu)^{\wedge}=f h\right)$;

(iii) $\lim _{t \rightarrow 0}\|\zeta * \exp (t \beta) * \eta-\zeta * \eta\|_{\infty}=0\left(\forall \zeta, \eta \in C^{\wedge}\right)$.

Proof. First suppose $\beta=\hat{\alpha}$ for some $\alpha \in \Lambda$. Then (i) follows from (22) and (ii) from Theorem 4 and Proposition 4. That (iii) holds follows from Propositions 4 and 11.

Now suppose that $\beta \in \bar{m}(\Sigma)$ satisfies (i), (ii), and (iii). From (i) follows that each $\exp (t \beta)$ is unitary; by Theorem 4 , it is in $G_{\hat{\gamma}}$. Thus there is a one-parameter subgroup $s$ of $G$ satisfying $\gamma(s(t))^{\wedge}=\exp (t \beta)$ for all $t \in R$. Condition (iii) just means that $\lim _{t \rightarrow 0} \gamma(s(t))^{\wedge}=\gamma(e)^{\wedge}$ in the quasi-strict topology. Thus, Proposition 11 implies that $s$ is continuous at 0 . Since $s$ is a homomorphism, it is continuous everywhere and so is an element of $\Gamma$. By Proposition 4, there exists $\alpha \in \Lambda$ such that $\gamma(s(t))=\exp (t \alpha)$ for all $t \in R$. Thus $\exp (t \beta)=\exp (t \alpha)^{\wedge}$ for all $t \in R$, which implies $\beta=\hat{\alpha}$ by Stone's theorem. Q.E.D.

\section{REFERENCES}

1. Charles A. Akemann and Gert K. Pedersen, Complications of semicontinuity in $C^{*}$-algebra theory, Duke Math. J. 40 (1973), 785-795. 1932.

2. S. Bochner, Vorlesungen über Fouriersche Integrale, Akademische Verlags Gesellschaft, Leipzig,

3. H. Boseck and G. Czichowski, Grundfunktionen und verallgemeinerte Funktionen auf Gruppen. I, Math. Nachr. 58 (1973), 215-240.

4. H. Boseck, Über Darstellungen lokal-kompakter topologischer Gruppen, Math. Nachr. 74 (1976), 233-251.

5. F. Bruhat, Distributions sur un groupe localement compact et applications à l'étude des représentations de groupes p-adiques, Bull. Soc. Math. France 89 (1961), 43-75.

6. C. Chevalley, Theory of Lie groups, Princeton Univ. Press, Princeton, N. J., 1946.

7. Nelson Dunford and Jacob T. Schwartz, Linear operators. Part II, Interscience, New York, 1963.

8. J. Dixmiers, Les $C^{*}$-algèbres et leurs représentations, Gauthier-Villars, Paris, 1969.

9. L__ Les algèbres d'operateurs dans l'espace Hilbertien, Gauthier-Villars, Paris, 1969.

10. P. Eymard, L'algèbre de Fourier d'un groupe localement compact, Bull. Soc. Math. France 92 (1964), 181-236. 
11. Edwin Hewitt and Kenneth A. Ross, Abstract harmonic analysis. Vol. I, Springer-Verlag, Berlin, 1963.

12. , Abstract harmonic analysis. Vol. II, Springer-Verlag, Berlin, 1970.

13. Herbert Heyer, Dualität lokalkompakter Gruppen, Lecture Notes in Math., vol. 150, SpringerVerlag, New York and Berlin, 1970.

14. G. Hochschild, The structure of Lie groups, Holden-Day, San Francisco, Calif., 1965.

15. R. Lashof, Lie algebras of locally compact groups, Pacific J. Math. 7 (1957), 1145-1162.

16. George W. Mackey, The Laplace transform for locally compact Abelian groups, Proc. Nat. Acad. Sci. U.S.A. 34 (1948), 156-162.

17. Kelly McKennon, The strict topology and the Cauchy structure of the spectrum of a C*-algebra, General Topology Appl. 5 (1975), 249-262.

18. The structure space of the trigonometric polynomials on a compact group, J. Reine Angew. Math. 307/308 (1979), 166-172.

19. D. Montgomery and L. Zippin, Topological transformation groups, Interscience, New York, 1955.

20. D. Raikov, Positive definite functions on commutative groups with an invariant measure, Dokl. Akad. Nauk SSSR (N.S.) 28 (1940), 296-300.

21. J. Riss, Éléments de calcul différentiel et théorie des distributions sur les groupes Abéliens localements compacts, Acta Math. 89 (1953), 45-105.

22. Walter Rudin, Functional analysis, McGraw-Hill, New York, 1973.

23. Masamichi Takesaki, Duality and von Neumann algebras, Lecture Notes in Math., vol. 247, Springer-Verlag, Berlin and New York, 1972.

24. _ Theory of operator algebras. I, Springer-Verlag, Berlin.

25. Martin Walter, $W^{*}$-algebras and non-Abelian harmonic analysis, J. Funct. Anal. 11 (1972), 17-38.

26. Garth Warner, Harmonic analysis on semi-simple Lie groups. I, Springer-Verlag, Berlin, 1972.

27. Andre Weil, L'intégration dans les groupes topologiques et ses applications, Hermann, Paris, 1941.

28. H. Yamabe, A generalization of a theorem of Gleason, Ann. of Math. (2) 50 (1953), 351-365.

Department of Mathematics, Washington State University, Pullman, Washington 99164 